\title{
Orientation-invariant transfer of training in the identification of rotated natural objects
}

\author{
JANICE E. MURRAY \\ University of Otago, Dunedin, New Zealand \\ PIERRE JOLICOEUR \\ University of Waterloo, Waterloo, Ontario, Canada \\ PATRICIA A. MCMULLEN \\ Dalhousie University, Halifax, Nova Scotia, Canada \\ and \\ MARGARET INGLETON \\ University of Waterloo, Waterloo, Ontario, Canada
}

\begin{abstract}
The effects of stimulus orientation on naming were examined in two experiments in which subjects identified line drawings of natural objects following practice with the objects at the same or different orientations. Half the rotated objects were viewed in the orientation that matched the earlier presentations, and half were viewed at an orientation that mismatched the earlier presentations. Systematic effects of orientation on naming time were found during the early presentations. These effects were reduced during later presentations, and the size of this reduction did not depend on the orientation in which the object had been seen originally. The results are consistent with a dual-systems model of object identification in which initially large effects of disorientation are the result of a normalization process such as mental rotation, and in which attenuation of the effects is due to a shift from the normalization system to a feature/part-based system.
\end{abstract}

Generally, our ability to identify objects successfully is not contingent upon viewing objects in a canonical orientation (typically, upright). However, the process of identifying objects that are disoriented is not entirely without consequence. The time to name disoriented line drawings of objects and animals increases systematically as a function of the amount of rotation of the object from the upright (Jolicoeur, 1985, 1988, 1990; Jolicoeur \& Milliken, 1989; Maki, 1986; McMullen \& Jolicoeur, 1992) for drawings rotated from $0^{\circ}$ to $120^{\circ}$. This pattern of naming times can be accounted for in the following manner, according to McMullen and Jolicoeur. In the long-term representation used to name drawings of objects, the relations among object parts are coded in terms of upright, internal object axes within a retinally aligned frame of reference. For a disoriented object to be recognized, the

This research was supported by grants from the Faculty of Graduate Studies and Research (Carleton University) and the University of Otago Research Committee to J.E.M., and awards from the Natural Sciences and Engineering Research Council of Canada to J.E.M. and P.J. P.A.M. was supported by ONR Contract NO014-89-J3016 to Martha Farah. We are grateful to Lise Paquet for the use of her equipment and to Paul Mohapel and Cynthia Finnie for assistance in conducting the experiments. We thank Michael Corballis, Ruth Maki, and an anonymous reviewer for their comments. Address reprint requests to J. E. Murray, Department of Psychology, University of Otago, Box 56, Dunedin, New Zealand (e-mail: jmur@otago.ac.nz). input representation has to be aligned with the stored representation through a normalization process (Ullman, 1989). Moreover, the normalization process underlying the linear portion of the naming function reflects the same analogue transformation process that is assumed to underlie the orientation effect observed in tasks requiring some judgement of left-right reflection-namely, mental rotation (Jolicoeur, 1985, 1988, 1990; Tarr \& Pinker, 1989).

The observed linear orientation effects on naming time are most pronounced when each drawing is viewed for the first time. With repeated exposure to the same objects, the magnitude of the orientation effect diminishes significantly (Jolicoeur, 1985, 1988; Jolicoeur \& Milliken, 1989; Maki, 1986; McMullen \& Jolicoeur, 1992). Furthermore, this practice effect is specific to the drawings presented repeatedly throughout the experiment (Jolicoeur, 1985). This suggests that subjects learn to minimize the effects of orientation in identifying disoriented objects they have seen previously, but do not learn how to identify disoriented objects in general.

Following repeated viewing, how are familiar disoriented line drawings recognized? One possibility is that observers may become more tuned to "orientation-free" information in a disoriented object as it becomes familiar. With increased familiarity comes increased sensitivity to orientation-invariant parts or attributes that could serve to reliably distinguish each particular object in the stimu- 
lus set (Jolicoeur, 1990; Jolicoeur, Snow, \& Murray, 1987). This would minimize the need for mental rotation (Takano, 1989).

Alternatively, the observer may continue to rely on orientation-specific representations of objects for identification. The reduced orientation effect could be a consequence of the formation of multiple representations of each familiar object, with a specific representation encoded in memory for each previously experienced orientation (Jolicoeur, 1985, 1988, 1990; Tarr \& Pinker, 1989). Mental rotation would still be used to identify an object following practice with a number of different orientations, but the amount of mental rotation required for one to align the input image with a stored representation would be reduced if the input representation of the object could be mentally rotated to the previously stored representation with the nearest orientation.

Tarr and Pinker (1989) provide compelling evidence for the viability of the multiple orientation-specific representations hypothesis. In four experiments, they presented subjects with three novel, letter-like, asymmetrical characters in a naming task. Following extended practice at naming the shapes at a small number of trained orientations, a significant reduction in the initially observed orientation effects was observed. More importantly, when subjects were presented with the same shapes at previously unseen orientations, response times were generally a function of the angular disparity from the closest learned orientation.

The stimuli used by Tarr and Pinker (1989) consisted of three very similar and highly confusable visual shapes that were specifically designed to be devoid of attributes that could serve as unique cues to identity. The visual characteristics of the stimuli may have induced the viewers to form multiple representations for subsequent identifications, because other alternative procedures such as extraction of orientation-invariant attributes were unavailable to them or perhaps simply too difficult to use. The many discriminations required in identifying objects in the natural environment frequently involve shapes less confusable than those used by Tarr and Pinker. Given the large and feature-rich stimulus set we must deal with in the real world, it is reasonable to speculate that alternative recognition strategies might be used. In this article we extend the findings of earlier work (e.g., Jolicoeur, 1985; Jolicoeur \& Milliken, 1989) and test the generality of Tarr and Pinker's findings by using a large, visually diverse set of natural objects and animals depicted in line drawings.

\section{EXPERIMENT 1}

In the first experiment, subjects in training named objects shown twice at the same orientation. This was expected to produce a robust practice effect manifested as a reduction in the effect of orientation on naming latency. Following this practice, subjects viewed half the objects in the orientation that matched the original orientation, and half in an orientation that mismatched the previous orientation. If recognition in this transfer phase is achieved by means of orientation-invariant representations, there should be no difference in naming latencies as a function of orientation for the objects viewed in the matched and mismatched orientations. On the other hand, if multiple orientation-specific representations are encoded in memory during practice, and if stimuli are rotated to the closest orientation, there should be a large effect of orientation on identification time for objects presented at mismatching orientations relative to objects presented at matching orientations.

\section{Method}

Subjects. Twenty-nine Carleton University undergraduates served as paid participants. The data from 5 subjects were eliminated for reasons outlined in the Results section. The final analyses were based on data from 24 subjects ( 10 males). All subjects were native speakers of English and had normal or corrected-to-normal vision. No subject had experience of the stimuli prior to the experiment.

Stimuli. The stimuli were 120 line drawings of natural objects and animals with a distinct top and bottom taken from Snodgrass and Vanderwart (1980). The accepted names for the drawings can be found in McMullen and Jolicoeur (1992). Each drawing was reduced and photocopied onto translucent Mylar film and then mounted in a $35-\mathrm{mm}$ slide frame at $60^{\circ}, 120^{\circ}, 240^{\circ}$, and $300^{\circ}$ of clockwise rotation in the picture plane. Drawings of an additional six objects were used in practice trials (hand, pineapple, pipe, tie, vest, and windmill).

Procedure. On each trial, a slide projector with an electronic shutter was used to show the subject a drawing on a rear-projection screen. The drawings subtended approximately $3^{\circ}$ of visual angle. The task was to name aloud the object or animal as quickly and appropriately as possible. Written instructions emphasized both speed and accuracy. The slide remained in view until the subject responded. Naming latency was measured from the onset of the shutter's opening to the onset of the subject's verbal response, as detected by a voice-activated relay. Response time was recorded to the nearest millisecond by an Apple Ile microcomputer that was also used to control stimulus presentation and to record response accuracy. Following each trial, the experimenter entered whether the response was correct or an error. Naming responses that did not correspond to any of the accepted names constituted errors. Trials spoiled by prenaming utterances or by the occasional spurious triggering of the voice-activated relay were also coded as errors. Two seconds after the response accuracy was entered, a beep was sounded by the computer. This indicated that the subject should initiate the next trial by pressing a key positioned in front of the subject. After every 60 trials there was a brief pause, during which the experimenter changed slide trays.

In the training phase of the experiment, the subjects received two presentations of a block of 120 trials. Within this block of trials, each of the 120 drawings was presented once. Thirty drawings were shown at each of the four orientations. The drawings within a block were randomly ordered, with the following constraint. Within a subblock of 24 items, each orientation was represented six times and there were no more than two consecutive occurrences of any particular orientation. To control for any object-specific effects on orientation, four sets of 120 trial blocks were created. Drawings appearing at one particular orientation in Set $\mathrm{A}$ appeared at the three remaining orientations in Sets B, C, and D, respectively. The subjects were randomly assigned to one set, with 6 subjects in each set. Thus, across the entire group of subjects, each drawing was presented equally often at each of the four orientations.

In the transfer phase of the experiment, each subject received one block of 120 trials. Each drawing shown in the training phase again appeared once at one of the four orientations. Half of the drawings 
were presented so that the orientation was the same as the original orientation (match transfer), with 15 drawings shown at each orientation. Each drawing in the other half was shown at one of the three orientations other than the one at which the drawing had been presented previously in the training phase (mismatch transfer). Of the 15 drawings presented at each of the four mismatching orientations, 5 were drawn from each of the three possible previous orientations.

The transfer trials were ordered randomly, with the constrain that within a subblock of 24 trials, there was an equal number of match and mismatch presentations at each of the four orientations. In addition, no more than two consecutive occurrences of any one orientation was allowed.

At the outset of the experiment, the subjects received six practice trials. These trials served to acquaint the subject with the experimental task and to permit any necessary adjustment of the volume control for the voice-activated relay. The entire session took approximately $1 \mathrm{~h}$.

\section{Results and Discussion}

To be included in the group of subjects used for statistical analysis, subjects had to obtain at least $80 \%$ correct responses at each orientation in each of the training-1, training-2, match-transfer, and mismatch-transfer conditions. Four subjects were rejected for failing to reach this criterion in the training phase. One subject's data could not be used because of voice-key malfunction.

For each subject, mean naming times and standard deviations for correct responses were calculated for each presentation condition and orientation, temporarily excluding the fastest and slowest reaction times. Any times that were greater or less than four standard deviations from this mean were rejected. Response times were rejected recursively on the basis of new cell means and standard deviations, until all the data satisfied this criterion. The fastest and slowest times of the last iteration were included in the final means, which are shown in Table 1 along with mean percent error rates. This procedure resulted in a rejection of $3.2 \%$ of the data. Following application of the outlier criterion, response times for clockwise and counterclockwise rotations of the same magnitude were averaged for the purposes of analysis. Thus, response times for objects presented at $60^{\circ}$ and $300^{\circ}$ were averaged to repre- sent $60^{\circ}$ departures from the upright, and, similarly, response times for objects presented at $120^{\circ}$ and $240^{\circ}$ were averaged to represent $120^{\circ}$ departures from the upright. For the initial analyses, response times for transfer items were also collapsed across previous orientation. Figure 1 shows the resulting mean naming latencies averaged across subjects and items, at each orientation $\left(60^{\circ}\right.$ or $120^{\circ}$ ) and presentation condition (training-1, training- 2 , match transfer, or mismatch transfer).

To investigate the effects of practice, a repeated measures analysis of variance (ANOVA) was performed on the data from the presentation conditions training-1, training-2, and match transfer, with presentation condition and orientation as factors. A significant main effect of presentation condition confirmed what is depicted in Figure 1, that naming time decreased with practice $[F(2,46)=56.1, p<.001]$. Naming time was also affected sharply by the degree of disorientation from the upright $[F(1,23)=57.7, p<.001]$. Furthermore, the effect of orientation diminished significantly over the three presentation conditions $[F(2,46)=9.6, p<.001]$. This interaction is important, because it indicates that three blocks of presentation were sufficient to reduce the effect of orientation. In fact, the effects of orientation were significantly diminished by the second training block of trials, as is indicated by a significant interaction in a comparison of training-1 and training- $2[F(1,23)=8.9, p<.01]$.

Of greatest interest with respect to the practice effect is a two-way ANOVA comparing the match- and mismatchtransfer conditions (i.e., objects that were shown in the training orientations vs. objects shown in new orientations during the transfer phase). A significant main effect of presentation condition revealed that objects that had been repeatedly viewed at one orientation were named faster than objects presented at a new orientation in Block 3 $[F(1,23)=31.5, p<.001]$. A dependence of naming time on orientation was also revealed $[(F(1,23)=20.3$, $p<.001]$. As would be expected from looking at Figure 1 , however, the interaction between presentation condition and orientation failed to reach significance $(F<1)$,

Table 1

Mean Response Times (RT, in Milliseconds) and Mean Percent Error (\%E) for Each Object at Each Orientation in the Training and Transfer Phases in Experiment 1

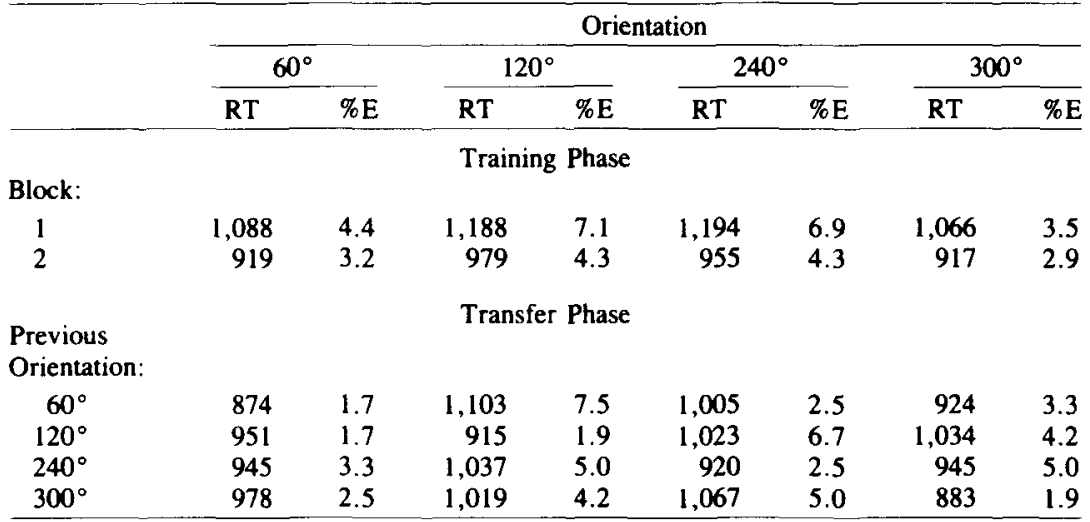

Note- $\mathrm{RT}$ and $\% \mathrm{E}$ for objects in the transfer phase are shown as a function of previous orientation in training. 


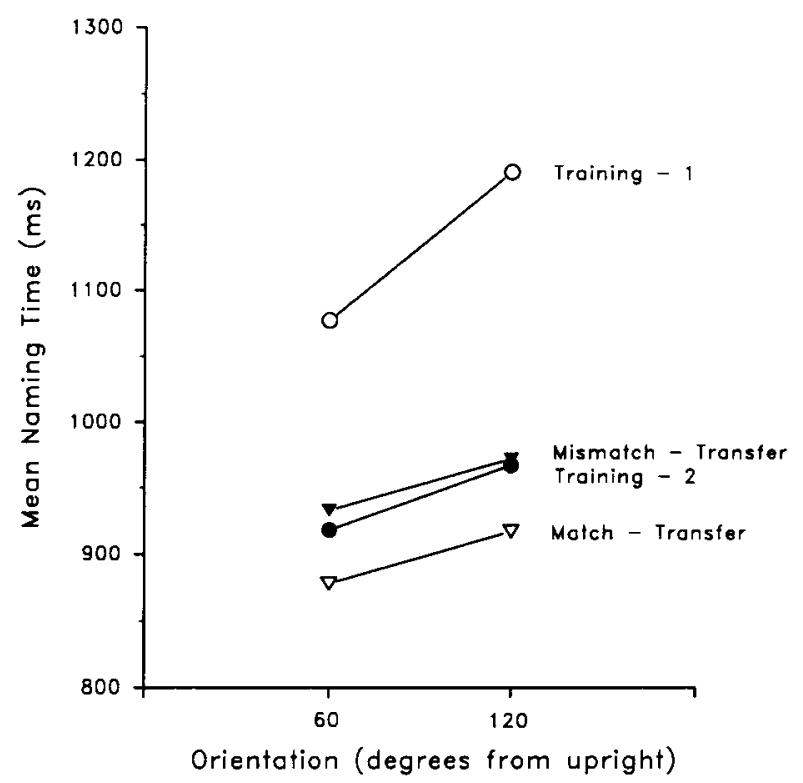

Figure 1. Mean naming time for training and match-, mismatchtransfer objects at each orientation in Experiment 1.

indicating that the effects of orientation did not differ across the two transfer conditions; the effect of practice did indeed transfer to new orientations.

A more specific comparison of the naming latencies was based on a direct prediction derived from the multiple orientation-specific representations hypothesis. The multiple representations view holds that recognition occurs by means of a transformation to the nearest orientationspecific representation encoded in memory. Accordingly, naming latencies to objects shown at $120^{\circ}$ should have been faster when the drawing had been viewed previously at $60^{\circ}$ (a $60^{\circ}$ distance between transfer and the nearest representation at $60^{\circ}$ ) than when the original orientation was $300^{\circ}$ (a $120^{\circ}$ distance between transfer and the nearest representation at $0^{\circ}$ ). Similarly, naming latencies to objects shown at $240^{\circ}$ should have been faster when the drawing had been viewed previously at $300^{\circ}\left(60^{\circ}\right.$ distance) than when the original orientation was $60^{\circ}\left(120^{\circ}\right.$ distance). The means shown in Table 1 clearly do not support this prediction. An ANOVA contrasting the two types of trials failed to support the difference expected on the basis of multiple orientation-specific representations hypothesis $[F(1,23)=1.6, p<.20]$. Instead, the results suggest that the practice effect did not depend on the orientation of the previously experienced object.

Similar analyses of error rates revealed a pattern of results that generally corresponded to the results of response times (the error analyses are available from the authors). None of these analyses suggests a speed-accuracy tradeoff explanation of the response time data.

The possibility that the reduced orientation effect following practice in naming natural stimuli is due to the formation and use of multiple orientation-specific repre- sentations was not supported in Experiment 1. There was no evidence to suggest that drawings were rotated to the nearest previously seen orientation. However, it is possible that such an explanation should not yet be discounted. Jolicoeur and Milliken (1989) have suggested that subjects may adopt a strategy of imagining and forming representations of drawings at a number of orientations in anticipation of being asked to name the same drawings at those orientations in later trials. In past studies (e.g., Jolicoeur, 1985; Jolicoeur \& Milliken, 1989), the development of such a strategy would have been possible, and it appears quite plausible, given that drawings seen at one orientation in a given block were seen at another orientation in a subsequent block. However, this strategy is not likely to have been used in Experiment 1, because a drawing was seen in only one orientation during the training phase. In spite of an absence of any inducement to store representations of each object at orientations other than the one experienced for that object, subjects may nevertheless have done so, given that the same four orientations were experienced repeatedly across the experiment. If subjects did form a representation of each object at all four possible orientations experienced in training, the results of Experiment 1 would have been obtained.

\section{EXPERIMENT 2}

Subjects in training named drawings presented at only two orientations-either $60^{\circ}$ and $120^{\circ}$ clockwise rotation or $60^{\circ}$ and $120^{\circ}$ counterclockwise rotation. At transfer, in addition to receiving the same two orientations experienced in training, the subjects received two surprise orientations. These surprise orientations involved rotations from the upright of the same magnitude as those orientations experienced in training but in the opposite direction. By virtue of having surprise transfer orientations that were completely novel and on a different side of the vertical axis, it is highly improbable that subjects would have formed imagined representations of drawings in training that would include the surprise orientations subsequently tested in transfer. The predictions relating to the multiple orientation-specific representations and orientationinvariant representations hypotheses are as in Experiment 1.

\section{Method}

Subjects. Sixteen (6 males) students of the University of Otago participated as paid subjects. All had normal or corrected-to-normal vision and were native speakers of English. No subject had any previous experience with the stimuli.

Stimuli and Apparatus. Eighty line drawings were selected from the set used in Experiment 1 . The names for the drawings are given in the Appendix. All the drawings were judged by the first author and an independent observer to be asymmetrical about any onedimensional axis. Stored images of the Snodgrass-Vanderwart drawings were created by scanning each drawing with an HPScanjet Plus. Each scanned drawing was then edited with PCPaintbrush software to produce uniform images for $60^{\circ}, 120^{\circ}, 240^{\circ}$, and $300^{\circ}$ of clockwise rotation. The drawings were presented on a Zenith 1492 VGA color monitor as black line drawings on a light gray background: they subtended approximately $3^{\circ}$ of visual angle. Stimulus presen- 
tation and collection of responses was controlled by an NEC Powermate 386 computer with Micro Experimental Laboratory software (Schneider, 1988). Naming latency was detected by a voice-activated relay connected to a microphone.

Procedure. On each trial, the subject viewed a small fixation cross at the center of the screen. Following a keypress by the subject, the screen was blanked for $1.5 \mathrm{sec}$, during which time the stimulus display was written to the screen. At the end of this interval, the screen was turned on at the top of the refresh cycle to reveal the drawing to the subject. The display remained on until the subject responded. As in the previous experiment, the subjects were instructed to name the drawing as quickly and accurately as possible. Naming latency was measured from the onset of the display screen to the onset of the subject's verbal response. Responses were detected by the voice-activated relay and recorded by the computer to the nearest millisecond. The trial concluded with the coding of the response as correct, incorrect, or spoiled by the experimenter.

In the training phase of the experiment, the subjects received four presentations of a block of 80 trials. A block consisted of a single presentation of each of the 80 drawings. Forty drawings were presented at $60^{\circ}$ of rotation in the picture plane, and 40 , at $120^{\circ}$. For half the subjects, the direction of rotation was clockwise $\left(60^{\circ}\right.$ and $120^{\circ}$ orientations), and for the other half, counterclockwise $\left(-60^{\circ}\right.$ and $-120^{\circ}$ orientations). Across the 16 subjects, each drawing was presented at each of the four orientations equally often.

In the fifth block of trials, the transfer phase, the same 80 drawings were presented once. For half the trials, the orientation of transfer was the same as the orientation of training (match transfer). Each drawing in the remaining half was shown at a new orientation, one not previously experienced (mismatch transfer). The degree of rotation of the two mismatch orientations from the upright corresponded to the degree of rotation of the match orientations, but with the direction of rotation of the mismatch drawings opposite that of the match drawings. Thus, for example, subjects who named drawings at $60^{\circ}$ and $120^{\circ}$ in training received drawings at $60^{\circ}$ and $120^{\circ}$ in the match-transfer condition and drawings at $-60^{\circ}$ and $-120^{\circ}$ in the mismatch-transfer condition. Twenty drawings were shown at each match and mismatch orientation. Trials in both the training and the transfer phases were randomly ordered. The experimental trials were preceded by six practice trials. The entire session took approximately $50 \mathrm{~min}$.

\section{Results and Discussion}

With the criterion of $80 \%$ correct responses having been met, the data from all 16 subjects were used for statistical analyses. Any response latencies less than $300 \mathrm{msec}$ or greater than $3,000 \mathrm{msec}$ were considered outliers. Application of this criterion resulted in a rejection of $.4 \%$ of the data. The mean response time for correct responses was calculated for each subject for each orientation within each training block and for each match and mismatch orientation in the transfer block. Response times for the transfer drawings were collapsed across previous orientation. Figure 2 shows the resulting mean latencies averaged across subjects and drawings at each orientation $\left(60^{\circ}\right.$ and $120^{\circ}$ from upright) for Block 1 training and match and mismatch transfer. The mean response times and error rates in the training phase of the experiment are presented in Table 2 .

The results from the transfer phase are presented first. A repeated measures ANOVA was performed on the means, with orientation and presentation condition (match or mismatch) as factors. Drawings named at the same orientation as that experienced during the previous four

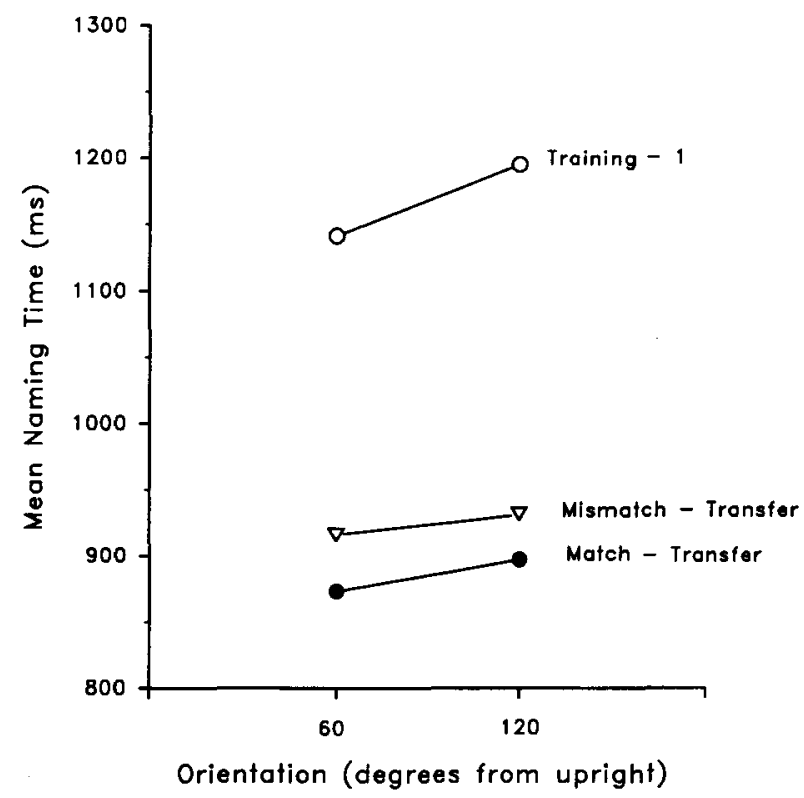

Figure 2. Mean naming time for training-1 and match-, mismatchtransfer objects at each orientation in Experiment 2.

blocks (match transfer) were responded to faster than those named at new orientations (mismatch transfer) $[F(1,15)=$ $6.0, p<.05$ ]. The effect of orientation did not reach significance $[F(1,15)=3.9, p<.10]$, nor did the interaction between presentation condition and orientation $(F<1)$. Thus, after repeated practice in naming drawings at the same orientations, response time is no longer affected by orientation. More importantly, this effect of practice is found equally for drawings presented at the original orientations and those presented at orientations never experienced previously in the task. In the latter case, these are orientations that were not experienced in the training phase, were not in the path of any postulated mental rotation, and were not likely to be anticipated by the subjects.

Table 2

Mean Response Times (RT, in Milliseconds) and Mean Percent Error (\%E) for Each Object in the Training and Transfer Phases in Experiment 2

\begin{tabular}{|c|c|c|c|c|}
\hline & \multicolumn{4}{|c|}{ Distance from Upright } \\
\hline & \multicolumn{2}{|c|}{$60^{\circ}$} & \multicolumn{2}{|c|}{$120^{\circ}$} \\
\hline & RT & $\% \mathrm{E}$ & RT & $\% \mathrm{E}$ \\
\hline \multicolumn{5}{|c|}{ Training Phase } \\
\hline \multicolumn{5}{|l|}{ Block: } \\
\hline 1 & 1,141 & 8.5 & 1,196 & 8.9 \\
\hline 2 & 984 & 4.3 & 1,040 & 5.0 \\
\hline 3 & 918 & 2.9 & 967 & 4.1 \\
\hline 4 & 881 & 3.6 & 909 & 3.4 \\
\hline \multicolumn{5}{|c|}{ Transfer Phase } \\
\hline \multicolumn{5}{|l|}{$\begin{array}{l}\text { Presentation: } \\
\text { Condition }\end{array}$} \\
\hline Match & 873 & 3.8 & 898 & 3.2 \\
\hline Mismatch & 916 & 4.4 & 932 & 3.5 \\
\hline
\end{tabular}

Note-RT and \%E are shown as a function of distance from the upright. 
To confirm the practice effect, the results from the first block of training were compared with those from the transfer block of trials, collapsing across match and mismatch conditions. An ANOVA with repeated measures on orientation and block ( 1 or 5$)$ revealed a significant effect of orientation $[F(1,15)=11.5, p<.01]$. A main effect of block confirmed that drawings experienced during Block 5 transfer were generally named faster than the same drawings presented in the first block of training $[F(1,15)=$ $54.0, p<.001]$. The predicted interaction between orientation and block was only marginally significant $[F(1,15)$ $=3.8, p<.07]$, but Bonferroni planned comparisons revealed the expected findings: The effect of orientation was significant for Block 1 training $(p<.05)$, but this was not the case for Block 5 transfer.

Error rates were analyzed in the same fashion as response times. In none of the analyses did orientation or the interaction between orientation and presentation condition reach statistical significance ( $F<1$ in all cases). The error analyses yielded nothing to suggest any speedaccuracy tradeoffs.

\section{GENERAL DISCUSSION}

In these experiments, the pronounced orientation effect on the time to name disoriented natural objects at initial viewing was increasingly attenuated following repeated exposure to the objects. The major finding derived from these studies is related to the lack of orientation specificity of this practice effect; the magnitude of the practice effect did not depend on the orientation in which the object had been seen originally.

These results are inconsistent with an account of the reduced orientation effect based on the transformation and comparison of orientation-specific representations. This type of recognition mechanism dictates that the attenuated orientation effect that accompanies objects named repeatedly at a particular orientation should not transfer to the same objects viewed at new orientations. The present data clearly show such a transfer. In addition, this transfer took place even when the novel orientations of the transfer phase were not experienced in training and could not have been anticipated. This makes less plausible the suggestion that the practice effect is the result of multiple orientation-specific representations formed following the imagining of rotated objects. Our results suggest that the reduction of the orientation effect for rotated natural objects is mediated by orientation-invariant representations.

The results are consistent with a dual-system or dualroute model of object identification in which the initially large effects of disorientation are the result of a timeconsuming normalization process, such as mental rotation (see Jolicoeur, 1990). Such a process is needed in order to maintain the spatial relations among parts or features so that those relations might be mapped onto relations stored in memory (see Koriat \& Norman, 1989; McMullen \& Jolicoeur, 1992). The diminution of the orientation effect is due to a shift from the alignment sys- tem to a feature- or part-based system following experience with the objects to be identified. What must be learned is which orientation-invariant parts or attributes, independently of their spatial relations, reliably permit discrimination among the objects in order to create and strengthen the appropriate representations. As suggested by Biederman (1987), one source of orientation-invariant parts may be geometric primitives called geons, derived from viewpoint-invariant nonaccidental properties of the stimulus. Local features and texture may also serve as sources of orientation-free information. The initial development of sensitivity to orientation-invariant information would be felt very rapidly (with as little as one viewing), but the shift from identification mediated by a mental-rotation system to identification completely mediated by a part-based system would likely be a gradual process requiring repeated exposure to the objects (Jolicoeur, 1990; Takano, 1989). The gradual decrease in the magnitude of the orientation effect with practice reported here and elsewhere (Jolicoeur, 1985, 1988; Jolicoeur \& Milliken, 1989; Maki, 1986; McMullen \& Jolicoeur, 1992) supports this hypothesis.

What happens if orientation-invariant information is not present or is difficult to isolate? The answer to this is suggested by the work of Tarr and Pinker (1989). It would appear that subjects make use of orientation-specific representations and continue to rely upon the mental-rotation system when the stimuli are distinguishable only by their two-dimensional spatial configurations. This would rarely be the case in the natural environment, where the large number of identifiable stimuli are diverse and rich in features such as texture and local features. As the present studies illustrate, under these conditions, viewers opt for a more feature/part-based system of recognition following practice. Taken together, these results and the work of Tarr and Pinker highlight the flexibility of the human pattern recognition system and point to the need for the development of multiple systems models.

The preceding account assumes that the basis of the difference between Tarr and Pinker's (1989) findings and the present results consists in the presence or absence of easily extracted orientation-invariant information. However, one other factor differed across the two sets of studies-namely, the number of stimuli to be identified. Tarr and Pinker presented three stimuli for identification. In our experiments, in addition to our using a set of objects that varied widely in features and their combination, the number of objects to be named was relatively large -120 in Experiment 1 and 80 in Experiment 2. It might be argued that set size is also a factor that influences the form of representation used to identify familiar objects. At the very least, forming and using orientation-specific representations would be easier with 3 stimuli than with 120 or 80 . Independently of the availability of orientationinvariant information in a stimulus, it may be more economical or efficient to form orientation-specific representations and continue to engage in mental rotation when set size is small than it is to determine which features consistently distinguish one object from others. Had we pre- 
sented small sets of natural stimuli, perhaps evidence for orientation-specific recognition would have been found. Although this is a possible argument, it does not have intuitive appeal. Consider the following three natural objects: a motorcycle, a sailboat, and a zebra. Following repeated experience with rotated versions of these objects, it seems highly likely that subjects would quickly learn that two circles (wheels), a triangle (sail) or black and white stripes could reliably permit one to discriminate among the three objects independently of orientation. The extraction of this type of information occurs rapidly in the early stages of visual processing (Biederman, 1987), and it would seem more efficient to use this orientationinvariant information than to continue to engage in timeconsuming transformation processes for mapping onto orientation-specific representations. Whether this is the case must be the subject of further research, because the present experiments do not allow us to rule out the possibility that set size influences the form of representation used in the identification of objects. But the present experiments do allow us to conclude that as individuals become increasingly familiar with a large set of visually diverse naturalistic objects, the recognition process used to identify these objects becomes orientation independent.

\section{REFERENCES}

Biederman, I. (1987). Recognition-by-components: A theory of human image understanding. Psychological Review, 94, 115-147.
Jolicoeur, P. (1985). The time to name disoriented natural objects. Memory \& Cognition, 13, 289-303.

Jolicoevr, P. (1988). Mental rotation and the identification of disoriented objects. Canadian Journal of Psychology, 42, 461-478.

Jolicoevr, P. (1990). On the role of mental rotation and feature extraction in the identification of disoriented objects: A dual-system theory. Mind \& Language, 5, 387-410.

Jolicoevr, P., \& Milliken, B. (1989). Identification of disoriented objects: Effects of context of prior presentation. Journal of Experimental Psychology: Learning, Memory, \& Cognition, 15, 200-210.

Jolicoeur, P., SNow, D., \& Murray, J. (1987). The time to identify disoriented letters: Effects of practice and font. Canadian Journal of Psychology, 41, 303-316.

Koriat, A., \& Norman, J. (1989). Why is word recognition impaired by disorientation while identification of single letters is not? Journal of Experimental Psychology: Learning, Memory, \& Cognition, 15 , 153-163.

MAKI, R. H. (1986). Naming and locating the tops of rotated pictures. Canadian Journal of Psychology, 40, 368-387.

McMullen, P. A., \& Jolicoeur, P. (1992). The reference frame and effects of orientation on finding the top of rotated objects. Journal of Experimental Psychology: Human Perception \& Performance, 18, 807-820.

SCHNEIDER, W. (1988). Micro Experimental Laboratory: An integrated system for IBM PC compatibles. Behavior Research Methods, Instruments, \& Computers, 20, 206-217.

Snodgrass, J. G., \& VANDERWART, M. (1980). A standardized set of 260 pictures: Norms for name agreement, image agreement, familiarity, and visual complexity. Journal of Experimental Psychology: Human Learning \& Memory, 6, 174-215.

Takano, Y. (1989). Perception of rotated forms: A theory of information types. Cognitive Psychology, 21, 1-59.

TARR, M. J., \& PINKer, S. (1989). Mental rotation and orientationdependence in shape recognition. Cognitive Psychology, 21, 233-282.

UllmaN, S. (1989). Aligning pictorial descriptions: An approach to object recognition. Cognition, 32, 193-254.

APPENDIX

Names of Snodgrass and Vanderwart Objects Used in Experiment 2

\begin{tabular}{llll}
\hline airplane & deer & kettle & rooster \\
alligator & dog & leopard & sailboat \\
ant & donkey & lion & seahorse \\
baby carriage & duck & monkey & seal \\
bam & eagle & motorcycle & sheep \\
bear & elephant & mouse & skunk \\
bee & fly & ostrich & sled \\
beetle & foot & peacock & spinning wheel \\
bicycle & football helmet & penguin & squirrel \\
bird & frying pan & piano & stove \\
boot & fox & pig & swan \\
bus & frog & pitcher & telephone \\
camel & giraffe & pot & tiger \\
car & goat & rabbit & tree \\
cat & gorilla & raccoon & train \\
caterpillar & grasshopper & record player & truck \\
church & harp & refrigerator & turtle \\
chicken & horse & rhinoceros & wagon \\
cow & ironing board & rocking chair & watering can \\
cup & kangaroo & roller skate & wishing well \\
\hline
\end{tabular}

(Manuscript received August 3, 1992;

revision accepted for publication March 5, 1993.) 